\title{
Mitochondrial alternative oxidase pathway acts as an electron sink during photosynthetic induction in Rumex K-1 leaves
}

\author{
D.D. CHENG ${ }^{*}$ and L.T. ZHANG ${ }^{* *+}$
}

College of Landscape Architecture and Forestry, Qingdao Agricultural University, Qingdao, Shandong, China* Institute of Oceanology, Chinese Academy of Sciences, Qingdao, Shandong, China**

\begin{abstract}
Chloroplast PSII photochemical efficiency is upregulated more rapidly than $\mathrm{CO}_{2}$ assimilation during photosynthesis induction, suggesting the existence of other electron sinks than that of $\mathrm{CO}_{2}$ assimilation. We hypothesized that the mitochondrial alternative oxidase (AOX) pathway could be such a sink. Inhibition of the AOX restricted light activation of the malate-oxaloacetate shuttle and caused an excessive reduction of PSI acceptor side and substantial accumulation of $\mathrm{Q}_{\mathrm{A}^{-}}$, hindering the photosynthetic linear electron transport rate (ETR) and leading to an imbalance between light energy absorption and exploitation during photosynthetic induction. ETR limitation also restricted the formation of thylakoid $\mathrm{pH}$ gradient, evidenced by a decreased de-epoxidation of the xanthophyll cycle, thus preventing nonphotochemical quenching. Delayed $\mathrm{CO}_{2}$ assimilation due to thylakoid $\mathrm{pH}$ gradient restriction was partially reversed by exogenous ATP application. The AOX pathway acts as a photosynthetic electron sink, protecting the photosynthetic apparatus against photoinhibition and accelerating the induction of $\mathrm{CO}_{2}$ assimilation during photosynthetic induction in Rumex K-1 leaves.
\end{abstract}

Keywords: alternative oxidase; photodamage; photosystem II photochemical efficiency; photosynthetic induction; Rumex K-1.

\section{Introduction}

Generally, when dark-adapted leaves are moved to light (photosynthetic induction), the stomata open within several minutes, although sometimes the process may take longer (Kirschbaum and Pearcy 1988). In addition, several key enzymes in the Calvin cycle are inactive in darkness and several minutes are required to activate them (Ruuska et al. 2000). Therefore, $\mathrm{CO}_{2}$ assimilation operates slowly in response to sudden illumination. Compared to
$\mathrm{CO}_{2}$ assimilation, PSII actual photochemical efficiency $\left(\Phi_{\text {PSII }}\right)$ is upregulated more rapidly during photosynthetic induction (Sui et al. 2011), indicating that, in addition to $\mathrm{CO}_{2}$ assimilation, alternative electron sinks contribute to rapid induction of photosynthetic linear electron flow after transfer from darkness to light. According to Makino et al. (2002) and Chen et al. (2004), $\Phi_{\mathrm{PSII}}$ and $\mathrm{CO}_{2}$ assimilation induction rates did not differ under low oxygen concentrations; therefore they suggested that the waterwater cycle is critical to rapid induction of photosynthetic

\section{Highlights}

- Inhibition of the Rumex $\mathrm{K}-1 \mathrm{AOX}$ delays $\mathrm{CO}_{2}$ assimilation

- $\mathrm{CO}_{2}$ assimilation is delayed due to restriction of thylakoid $\Delta \mathrm{pH}$ generation

- The Rumex K-1 AOX pathway acts as an electron sink during photosynthetic induction
Received 10 March 2021

Accepted 5 October 2021

Published online 23 November 2021

Corresponding author

e-mail: zhanglitao666@163.com

Abbreviations: ABS/RC - light absorption per reaction center; AOX - alternative oxidase; COX - cytochrome oxidase; ETR - linear electron transport rate; NADP-MDH - NADP-malate dehydrogenase; NPQ - nonphotochemical quenching; OAA - oxaloacetate; PRI - photochemical reflectance index; $\mathrm{q}_{\mathrm{E}}$ - fast component of NPQ; $\mathrm{q}_{\mathrm{P}}$ - photochemical quenching; ROS - reactive oxygen species; SHAM - salicylhydroxamic acid; $\mathrm{TR}_{0} / \mathrm{RC}$ - light trapping per reaction center; $\Phi_{\mathrm{PSII}}$ - actual photochemical efficiency of PSII; $\Psi_{0}$ - excitation efficiency for electron transport beyond $\mathrm{Q}_{\mathrm{A}}$.

Acknowledgements: Scientific Research Foundation for Advanced Scholars, Qingdao Agricultural University (20210056), and State Key Laboratory of Crop Biology (2017KF05) financed this study.

Conflict of interest: The authors declare that they have no conflict of interest. 
linear electron flow. However, Driever and Baker (2011) reported that no significant water-water cycle activity was detected during photosynthetic induction in maize leaves, although linear electron flux was higher than that required for $\mathrm{CO}_{2}$ assimilation. Moreover, treatment with a low concentration of oxygen suppresses both the waterwater cycle and mitochondrial respiration (Spicer and Holbrook 2007, Zabalza et al. 2009). Thus, the function of the water-water cycle during photosynthetic induction may be overestimated by Makino et al. (2002) and Chen et al. (2004).

Over the past two decades, interactions between respiration and photosynthesis have attracted increased attention (Sweetlove et al. 2006, Noguchi and Yoshida 2008, Dahal et al. 2014, 2015; Ogawa and Sonoike 2015, Florez-Sarasa et al. 2016, Zhang et al. 2017, Del-Saz et al. 2018, Hu et al. 2018, Mathur et al. 2018). Several critical metabolic pathways (e.g., nitrogen assimilation and photorespiration), require reactions occurring in both chloroplasts and mitochondria (Betti et al. 2016). In addition, excessive reducing equivalents generated as (reduced) nicotinamide adenine dinucleotide phosphate (NADPH) by photosynthesis can be transported to mitochondria via mechanisms such as the malateoxaloacetate (-OAA) shuttle and then oxidized via the respiratory electron transport chain (Noguchi and Yoshida 2008).

In higher plants, mitochondrial electron transport occurs via two pathways: the cyanide-sensitive cytochrome oxidase (COX) pathway and the cyanideinsensitive alternative oxidase (AOX) pathway (Miller et al. 2011). The COX pathway generates a proton gradient to produce the majority of ATP in mitochondria, whereas the AOX pathway is nonphosphorylating and can oxidize reducing equivalents efficiently, without being limited by the proton gradient across the mitochondrial inner membrane or the cellular ATP/ADP ratio (Yoshida et al. 2007). Several studies have indicated that when the AOX pathway is inhibited, the photochemical efficiency or the photosynthetic rate is reduced (Dinakar et al. 2010a,b; Del-Saz et al. 2018). Moreover, Yoshida et al. (2007) suggested that the AOX pathway is upregulated to oxidize excessive reducing equivalents generated by photosynthesis in Arabidopsis. Florez-Sarasa et al. (2016) suggested that AOX activity in vivo can act as a sink for the excess of electrons from the chloroplast, probably via photorespiratory glycine oxidation, thus improving photosynthetic performance when AOX activity is not induced under severe high-light stress. Watanabe et al. (2016) suggested that mitochondrial AOX pathwayassociated photoprotection of PSII is related to the photorespiratory pathway. Sunil et al. (2019) indicated that photorespiration is complemented by the AOX pathway to optimize photosynthesis and protect against abiotic stress. Dahal and Vanlerberghe (2017) suggested that the AOX pathway maintains both mitochondrial and chloroplast function during drought. Furthermore, Del-Saz et al. (2018) reviewed that the AOX pathway provides flexibility in cellular energy and carbon metabolism under stress conditions. Thus, the mitochondrial
AOX pathway may benefit photosynthesis by dissipating excess reducing equivalents generated in chloroplasts and protecting the photosynthetic mechanism against photoinhibition during steady-state photosynthesis. Nevertheless, it remains unclear, whether the rapid response of photosynthetic linear electron flow to sudden illumination during photosynthetic induction is partially dependent on the mitochondrial AOX pathway.

NADP-malate dehydrogenase (NADP-MDH, EC 1.1.1.82) is a critical enzyme in the malate-OAA shuttle and the major mechanism for the transfer of excess reducing equivalents from chloroplasts to mitochondria; the reducing equivalents are then oxidized via the AOX pathway (Yokochi et al. 2021). Activation of NADP-MDH increases rapidly to almost $80 \%$ of full activation after only $1 \mathrm{~min}$ of illumination (Padmasree and Raghavendra 2001). In addition, other experiments revealed that $25-35 \%$ of mitochondrial respiration occurs through AOX, even without light, and the AOX pathway can readily and inexpensively respond to environmental fluctuations (e.g., light) (Noguchi and Yoshida 2008, Florez-Sarasa et al. 2009, Rasmusson et al. 2009, Dinakar et al. 2010b, Yoshida et al. 2011). Therefore, we hypothesized that the AOX pathway also acts as a sink for electrons generated by photosynthesis during photosynthetic induction in Rumex K-1 leaves. If so, the following question is raised: Which are the physiological functions of rapid induction of AOX pathway-dependent photosynthetic linear electron flow during photosynthetic induction in Rumex K-1 leaves?

\section{Materials and methods}

Plant material and treatments: This study was conducted in a growth cabinet of Shandong Agriculture University $\left(36^{\circ} 11^{\prime} \mathrm{N}, 117^{\circ} 7^{\prime} \mathrm{E}\right)$. Rumex K-1 (a hybrid of Rumex patientia and Rumex tianschaious) is a fast-growing, highyield, excellent quality (high protein), easy to cultivate fodder crop. In China, Rumex K-1 widely grows in the understory since it has strong adaptability to changes in the environment (Omarova et al. 1998, Li 2000). Rumex $\mathrm{K}-1$ plants were grown from seeds in pots $(20 \mathrm{~cm}$ diameter, $30 \mathrm{~cm}$ height) with rich soil under a 14-h photoperiod [600 $\mu$ mol(photon) $\left.\mathrm{m}^{-2} \mathrm{~s}^{-1}\right]$ at $22 / 18^{\circ} \mathrm{C}$ (day/night). Plants were reduced to one plant per pot after two weeks from sowing. Sufficient water and nutrients were provided throughout to avoid drought and nutrient stress. Experiments commenced four weeks after germination just before the emergence of leaf six; the third true leaf was used in this study.

Intact chloroplasts were extracted following the protocol of Bartoli et al. (2005). Leaves (10 g) were ground in buffer supplemented with $50 \mathrm{mM}$ 4-(2-hydroxyethyl)1-piperazine ethane sulphonic acid (HEPES)-KOH (pH 7.5), $330 \mathrm{mM}$ sorbitol, $2 \mathrm{mM} \mathrm{Na}_{2} \mathrm{EDTA}_{1} 1 \mathrm{mM} \mathrm{MgCl}_{2}$, $5 \mathrm{mM}$ ascorbic acid, and $0.05 \%(\mathrm{w} / \mathrm{v})$ bovine serum albumin, using a hand-held homogenizer. Homogenates were filtered through $20-\mu \mathrm{m}$ nylon mesh, followed by centrifugation at $3,000 \times g$ for $5 \mathrm{~min}$ at $4^{\circ} \mathrm{C}$. Pellets were suspended in wash medium (50 mM HEPES-KOH, pH 
7.5, $330 \mathrm{mM}$ sorbitol) and loaded into a 35\% (v/v) Percoll solution in wash medium, followed by centrifugation at $2,500 \times \mathrm{g}$ for $5 \mathrm{~min}$ at $4^{\circ} \mathrm{C}$. Of the isolated chloroplasts, $75 \%$ were intact, as determined by ferricyanide-dependent $\mathrm{O}_{2}$ evolution (Walker 1988) measured using an oxygen electrode (Oxylab-2, Hansatech, King's Lynn, UK). The pellet containing intact chloroplasts was used to measure modulated chlorophyll $(\mathrm{Chl})$ fluorescence in the presence of $0,0.2,0.6$, or $1 \mathrm{mM}$ salicylhydroxamic acid (SHAM). SHAM is a relatively selective competitive inhibitor of the AOX (Laties 1982, Chivasa and Carr 1998, Murphy et al. 1999, Fisher et al. 2020). AOX pathway capacity was measured using an oxygen electrode (Oxylab-2, Hansatech, King's Lynn, UK) after adding KCN solution ( $3 \mathrm{mM}$ ) to the assay buffer. The AOX pathway capacity was defined as $\mathrm{O}_{2}$ uptake in the presence of $\mathrm{KCN}$ (Robson and Vanlerberghe 2002).

Leaves of Rumex K-1 were excised and immediately placed in a humidified insulated chest and transported to the laboratory within $10 \mathrm{~min}$, where the petioles were recut underwater to maintain hydraulic conductance in the leaf xylem vessels (Heber et al. 1986, Scoffoni and Sack 2015). Each three leaves were dipped into 50-ml Erlenmeyer flasks (pyrex glass) containing 0 (control), $0.2,0.6$, or $1 \mathrm{mM}$ SHAM, respectively. Further, three leaves were dipped into equally sized tubes containing water (0 mM SHAM, control), $1 \mathrm{mM} \mathrm{SHAM,} \mathrm{or} 1 \mathrm{mM}$ SHAM $+0.5 \mathrm{mM}$ ATP. The concentrations of SHAM used were sufficiently low to avoid any possible side effects on photosynthetic machinery (Diethelm et al. 1990). Leaves were allowed to absorb the different solutions for $5 \mathrm{~h}$ under weak irradiance $\left[20 \mu \mathrm{mol}\right.$ (photon) $\mathrm{m}^{-2} \mathrm{~s}^{-1}$ ]. The irradiance was conducted under a microwave sulfur lamp (MSL1000N1, Ningbo, China), which is a kind of efficient full spectrum electrodeless lamp. Furthermore, $0.5 \mathrm{mM}$ ATP was resupplied to leaves in the SHAM + ATP treatment at 2 and $0.5 \mathrm{~h}$ before measurements. For each experiment, 20 leaf discs $\left(0.5 \mathrm{~cm}^{2}\right)$ were obtained from three leaves; 10 leaf discs were punched symmetrically along the vein of each leaf, then the 30 discs were mixed and 20 discs were randomly chosen for use. At least three replicates were conducted for each experiment.

Enzyme assays: NADP-MDH in the soluble fraction of homogenate obtained as described above was extracted and assayed according to Dutilleul et al. (2003). Fifteen leaf discs $\left(0.5 \mathrm{~cm}^{2}\right)$ were ground in liquid $\mathrm{N}_{2}$ with a mortar and pestle and then extracted with $50 \mathrm{mM}$ HEPES-KOH buffer ( $\mathrm{pH}$ 7.5) supplemented with $10 \mathrm{mM} \mathrm{MgCl}_{2}, 1 \mathrm{mM}$ $\mathrm{Na}_{2}$ EDTA, $5 \mathrm{mM}$ dithiothreitol (DTT), a protease inhibitor tablet, $5 \%(\mathrm{w} / \mathrm{v})$ insoluble polyvinylpyrrolidone, and $0.05 \%(\mathrm{v} / \mathrm{v})$ Triton $X-100$. After centrifugation for $5 \mathrm{~min}$ at $14,000 \times g$ at $4^{\circ} \mathrm{C}$, enzyme activity in supernatants was determined using a $U V-2550$ spectrophotometer (Shimadzu, Kyoto, Japan). Assays were conducted in $40 \mathrm{mM}$ Tricine-KOH (pH 8.3), $150 \mathrm{mM} \mathrm{KCl,} 1 \mathrm{mM}$ $\mathrm{Na}_{2}$ EDTA, $5 \mathrm{mM}$ DTT, $0.2 \mathrm{mM}$ NADPH, and $2 \mathrm{mM}$ OAA.

$\mathbf{P}_{700}$ redox state: The redox state of $\mathrm{P}_{700}$ was measured following a previously described protocol (Yoshida et al.
2007) using a PEA Senior instrument (Hansatech Instruments) as the emitter-detector unit. An array of four $650-\mathrm{nm}$ light-emitting diodes (LED, peak $650 \mathrm{~nm}$ ) generated red light at $1,000 \mu \mathrm{mol}$ (photon) $\mathrm{m}^{-2} \mathrm{~s}^{-1}$, and an OD820 LED (Opto Diode Corp., Newbury Park, CA, USA) generated the modulated $(33.3 \mathrm{kHz})$ far-red light for measurement at $820 \mathrm{~nm}$. The $\mathrm{P}_{700}$ oxidation ratio was calculated as $\Delta \mathrm{A} / \Delta \mathrm{A}_{\max }$, where $\Delta \mathrm{A}$ and $\Delta \mathrm{A}_{\max }$ are the in vivo absorbances at $820 \mathrm{~nm}$ under red light and far-red light illumination, respectively.

Chlorophyll (Chl) a fluorescence transients (OJIP transients): Leaf OJIP transients were measured during photosynthetic induction using a Handy Plant Efficiency Analyzer (Handy PEA, Hansatech Instruments, Norfolk, UK). Red light at $1,000 \mu \mathrm{mol}$ (photon) $\mathrm{m}^{-2} \mathrm{~s}^{-1}$ provided by an array of three LED (peak $650 \mathrm{~nm}$ ) caused a reduction in the photosynthetic electron transport chain during photosynthetic induction. OJIP transients were measured during photosynthetic induction under the red light of $3,000 \mu \mathrm{mol}$ (photon) $\mathrm{m}^{-2} \mathrm{~s}^{-1}$.

Using JIP-test (Strasser and Strasser 1995, Smit et al. 2009, Yusuf et al. 2010), OJIP transients were analyzed based on the following original data: (1) fluorescence intensity, $\mathrm{F}_{0}$, at $50 \mu \mathrm{s}$, when all PSII reaction centers (RCs) were open; (2) maximal fluorescence intensity, $F_{m}$, based on excitation intensity, assumed to be sufficiently high to close all PSII RCs; (3) fluorescence intensities at $2 \mathrm{~ms}$ (J-step) and $300 \mu \mathrm{s}$. The following parameters were used to quantify PSII behavior: (1) the efficiency with which a trapped exciton was able to move an electron into the electron transport chain further than $\mathrm{Q}_{\mathrm{A}}^{-}$(the excitation efficiency for electron transport beyond $\left.\mathrm{Q}_{\mathrm{A}}^{-}\right)$: $\Psi_{0}=$ $1-\mathrm{V}_{\mathrm{J}}$, where $\mathrm{V}_{\mathrm{J}}=\left(\mathrm{F}_{2 \mathrm{~ms}}-\mathrm{F}_{0}\right) /\left(\mathrm{F}_{\mathrm{m}}-\mathrm{F}_{0}\right)$; (2) the specific energy fluxes (per RC) to achieve light energy absorption $(\mathrm{ABS} / \mathrm{RC})$ and trapping $\left(\mathrm{TR}_{0} / \mathrm{RC}\right)$.

Chl fluorescence parameters: An $F M S-2$ pulse-modulated fluorometer (Hansatech Instruments, Norfolk, UK) was used to measure $\mathrm{Chl}$ fluorescence parameters of leaves according to the method of Chen et al. (2007). Maximal fluorescence $\left(\mathrm{F}_{\mathrm{m}}\right)$ was measured under a $0.8-\mathrm{s}$ saturating light of $8,000 \mu \mathrm{mol}$ (photon) $\mathrm{m}^{-2} \mathrm{~s}^{-1}$ on a darkadapted leaf disc. Then, actinic light of $1,000 \mu \mathrm{mol}$ (photon) $\mathrm{m}^{-2} \mathrm{~s}^{-1}$ was provided by an FMS-2 light source, and Chl fluorescence induction or relaxation curves were recorded. Subsequently, steady-state fluorescence $\left(\mathrm{F}_{\mathrm{S}}\right)$ was recorded, and a second 0.8 -s saturating light of $8,000 \mu \mathrm{mol}$ (photon) $\mathrm{m}^{-2} \mathrm{~s}^{-1}$ was set to determine maximal fluorescence in the light-adapted state $\left(\mathrm{F}_{\mathrm{m}}{ }^{\prime}\right)$. Then, actinic light was turned off, and the minimal fluorescence in the light-adapted state $\left(\mathrm{F}_{0}{ }^{\prime}\right)$ was measured using a 3-s illumination with far-red light.

An FMS-2 pulse-modulated fluorometer (Oxylab-2, Hansatech Instruments, Norfolk, UK), based on a Clarktype oxygen electrode, was used to determine the $\mathrm{Chl}$ fluorescence parameters of intact Rumex K-1 chloroplasts. After $10 \mathrm{~min}$ of dark incubation, actinic light $[1,000$ $\mu \mathrm{mol}\left(\right.$ photon) $\mathrm{m}^{-2} \mathrm{~s}^{-1}$ ] was supplied by the Oxylab-2 light source. $\mathrm{F}_{\mathrm{m}}$ was determined during the dark incubation. Following the onset of actinic light irradiation, $\mathrm{F}_{\mathrm{S}}, \mathrm{F}_{\mathrm{m}}$, 
and $\mathrm{F}_{0}{ }^{\prime}$ were recorded at various time points during photosynthetic induction.

The following parameters were obtained: (1) the photochemical quenching coefficient, $\mathrm{q}_{\mathrm{P}}=\left(\mathrm{F}_{\mathrm{m}}{ }^{\prime}-\mathrm{F}_{\mathrm{S}}\right) /\left(\mathrm{F}_{\mathrm{m}}{ }^{\prime}-\mathrm{F}_{0}{ }^{\prime}\right)$, and the proportion of closed PSII RCs, $1-\mathrm{q}_{\mathrm{P}}$; (2) the linear electron transport rate $(\mathrm{ETR})=0.84 \times 0.5 \times \Phi_{\mathrm{PSII}} \times \mathrm{PPFD}$, $\Phi_{\mathrm{PSII}}=1-\mathrm{F}_{\mathrm{S}} / \mathrm{F}_{\mathrm{m}}{ }^{\prime}$; and (3) nonphotochemical quenching, $\mathrm{NPQ}=\left(\mathrm{F}_{\mathrm{m}}-\mathrm{F}_{\mathrm{m}}{ }^{\prime}\right) / \mathrm{F}_{\mathrm{m}}{ }^{\prime}$.

De-epoxidation of the xanthophyll cycle: A Unispec portable spectrometer, with bifurcated fiber optics and a leaf clip (PP Systems, Amesbury, MA, USA), was used to measure de-epoxidation of the xanthophyll cycle, denoted by the photochemical reflectance index $(\Delta \mathrm{PRI})$ of leaves during photosynthetic induction. Fiber optics were held with a leaf clip at a $60^{\circ}$ angle to a leaf disc. Leaf irradiation was conducted at approximately 1,000 $\mu$ mol(photon) $\mathrm{m}^{-2} \mathrm{~s}^{-1}$ via one side of the bifurcated fiber from the halogen lamp in the spectrometer. The leaf photochemical reflectance index (PRI) was calculated from the following reflectance data (Gamon and Surfus 1999): PRI $=\left(R_{531}-\right.$ $\left.\mathrm{R}_{570}\right) /\left(\mathrm{R}_{531}+\mathrm{R}_{570}\right)$, where $\mathrm{R}$ denotes reflectance and the subscript is the wavelength in nanometers. Furthermore, the de-epoxidation of the xanthophyll cycle was calculated

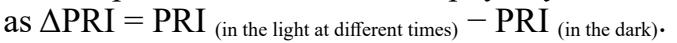

$\mathrm{CO}_{2}$ assimilation rate: A CIRAS-2 instrument (PP Systems, Amesbury, MA, USA) was used to measure the rate of induction of $\mathrm{CO}_{2}$ assimilation rate in leaves at $25^{\circ} \mathrm{C}$ and $360 \mu \mathrm{mol} \mathrm{mol}{ }^{-1} \mathrm{CO}_{2}$, following the method of Chen et al. (2007). Dark-adapted leaves were inserted into a CIRAS-2 leaf cuvette and then illuminated under actinic light at $1,000 \mu \mathrm{mol}$ (photon) $\mathrm{m}^{-2} \mathrm{~s}^{-1}$ provided by CIRAS-2 light source.

Chemicals: All the compounds used in this study were manufactured by Sigma (St. Louis, MO, USA) except where otherwise specified.

Statistical analyses: Results are presented as the mean \pm SE of at least three independent measurements. To evaluate the differences in parameters determined at different concentrations of SHAM, an LSD (least significant difference) test was performed in SPSS software version 16.0 (SPSS Inc., Chicago, IL, USA) with a 5\% significance level.

\section{Results}

Our results showed no significant difference in $\Phi_{\mathrm{PSII}}, \mathrm{q}_{\mathrm{P}}$, or NPQ during photosynthetic induction after SHAM treatment of Rumex K-1 leaves (Fig. 1). Further, there were no significant differences in $\mathrm{Chl} a$ fluorescence transients or other relevant parameters (e.g., $\mathrm{F}_{0}$ and $\left.\mathrm{F}_{\mathrm{m}}\right)$ after SHAM treatment of Rumex K-1 leaves in the dark (Fig. 1S, supplement). These results indicate that the concentrations of SHAM used in this study had no direct effects on the photosynthetic machinery.

In control samples, the NADP-MDH activation state increased rapidly to almost $75 \%$ full activation after only 1 min of exposure to irradiation (Fig. $2 A$ ). SHAM treatment did not significantly affect NADP-MDH activity in the dark. However, SHAM treatment significantly suppressed the light activation of NADP-MDH after 1 min during photosynthetic induction of Rumex K-1 leaves and the extent of light activation of NADP-MDH was remarkably downregulated with increasing SHAM concentration (Fig. 2A). Compared with controls, NADP$\mathrm{MDH}$ activation state was suppressed by 16.3, 27.7, and $37.4 \%$ following treatment with $0.2,0.6$, and $1 \mathrm{mM}$ SHAM at 10 -min exposure to irradiation, respectively (Fig. 2A). To determine the stromal redox state, $\mathrm{P}_{700}(\mathrm{RCs}$ of PSI) redox state in SHAM-treated leaves was monitored during photosynthetic induction. SHAM treatment led to a significant reduction in the $\mathrm{P}_{700}$ oxidation ratio, and the extent of $\mathrm{P}_{700}$ oxidation decreased with increasing SHAM concentration during photosynthetic induction. Compared with controls, the $\mathrm{P}_{700}$ oxidation ratio was suppressed by $29.7,44.2$, and $65.2 \%$ following treatment with $0.2,0.6$, and $1 \mathrm{mM}$ SHAM treatment at 4-min of exposure to irradiation, respectively (Fig. $2 B$ ).

To examine the redox state of the PSII acceptor side,

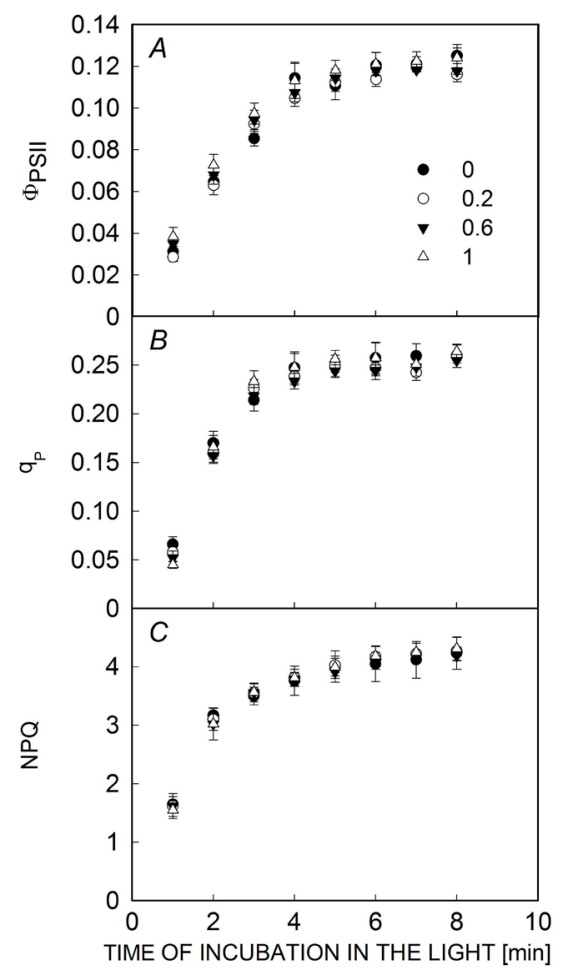

Fig. 1. (A) Photosystem II actual photochemical efficiency $\left(\Phi_{\mathrm{PSII}}\right),(B)$ photochemical quenching coefficient $\left(\mathrm{q}_{\mathrm{P}}\right)$, and (C) nonphotochemical quenching (NPQ) in isolated Rumex $\mathrm{K}-1$ chloroplasts in the presence of 0 (control), 0.2, 0.6 , or $1 \mathrm{mM}$ salicylhydroxamic acid (SHAM) during photosynthetic induction. Chloroplasts treated with or without SHAM were incubated without light for $10 \mathrm{~min}$ and then irradiated with $1,000 \mu$ mol(photon) $\mathrm{m}^{-2} \mathrm{~s}^{-1}$ for different periods of time. Values are the mean $\pm \mathrm{SE}, n=5$. The significance (5\% level) between groups was evaluated using the LSD test. 


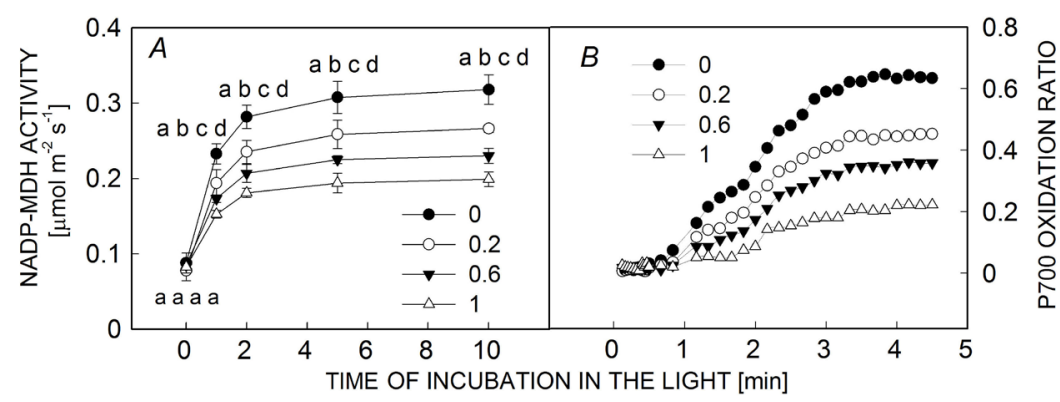

Fig. 2. (A) NADP-malate dehydrogenase (NADP-MDH) activity and $(B) \quad \mathrm{P}_{700}$ oxidation ratio in Rumex $\mathrm{K}-1$ leaves in the presence of 0 (control), $0.2,0.6$, or $1 \mathrm{mM}$ SHAM during photosynthetic induction. Rumex K-1 leaves with or without SHAM treatments were exposed to light at 1,000 $\mu \mathrm{mol}\left(\right.$ photon) $\mathrm{m}^{-2} \mathrm{~s}^{-1}$ for different periods of time. The lowercase letters represent values that differed significantly in the LSD range test $(P<0.05)$. Values are mean $\pm \mathrm{SE}, n=5$.
OJIP transients were measured in Rumex K-1 leaves during photosynthetic induction. The results showed that $\Psi_{0}$ increased progressively during photosynthetic induction while SHAM treatment suppressed the increase in $\Psi_{0}$ after illumination (Fig. $3 A$ ). The change in photosynthetic linear ETR kinetics after SHAM treatment was similar to that in $\Psi_{0}$ during photosynthetic induction in Rumex K-1 leaves (Fig. 3B). The proportion of closed PSII RCs $\left(1-\mathrm{q}_{\mathrm{P}}\right)$ decreased gradually after illumination in control and SHAM treatment significantly limited the decrease of $1-\mathrm{q}_{\mathrm{p}}$ during photosynthetic induction (Fig. 3C). There were no significant changes in light absorption per $\mathrm{RC}(\mathrm{ABS} / \mathrm{RC})$ or light trapped per $\mathrm{RC}\left(\mathrm{TR}_{0} / \mathrm{RC}\right)$ after illumination. Further, SHAM treatment did not cause any significant difference in the $\mathrm{ABS} / \mathrm{RC}$ or $\mathrm{TR}_{0} / \mathrm{RC}$ during photosynthetic induction (Fig. 4).

In controls, de-epoxidation of the xanthophyll cycle expressed as $\triangle$ PRI increased progressively after illumination, while SHAM treatment limited this increase (Fig. 5A). NPQ was formed rapidly after illumination and its formation was limited by SHAM treatment during photosynthetic induction in Rumex K-1 leaves (Fig. 5B). Furthermore, NPQ imaging of leaf discs infiltrated with SHAM showed similar results during photosynthetic induction (Fig. 2S, supplement). When the leaves were transferred from light to darkness, NPQ was promptly quenched (within $1 \mathrm{~min}$ ) and the differences in NPQ level between leaves treated with different concentrations of SHAM were eliminated (Fig. 5B).

To determine whether the mitochondrial AOX affected the rate of activation of Calvin cycle enzymes during photosynthetic induction, $\mathrm{CO}_{2}$ assimilation rate induction curves in Rumex $\mathrm{K}$-1 leaves were plotted in the presence of water (control), SHAM, or SHAM + ATP. The results showed that the $\mathrm{CO}_{2}$ assimilation rate responded more slowly to sudden illumination in SHAM-treated leaves compared with that in controls. The most significant difference (44.7\% decrease) in $\mathrm{CO}_{2}$ assimilation rate was after $14 \mathrm{~min}$ of exposure to irradiation in SHAMtreated leaves compared with that in controls. The induction of $\mathrm{CO}_{2}$ assimilation in SHAM-treated leaves during photosynthetic induction was partially recovered by exogenous application of ATP, which recovered $\mathrm{CO}_{2}$ assimilation to a $23.3 \%$ decrease in SHAM-treated leaves compared with that in controls at $14 \mathrm{~min}$ of exposure to irradiation (Fig. 6).

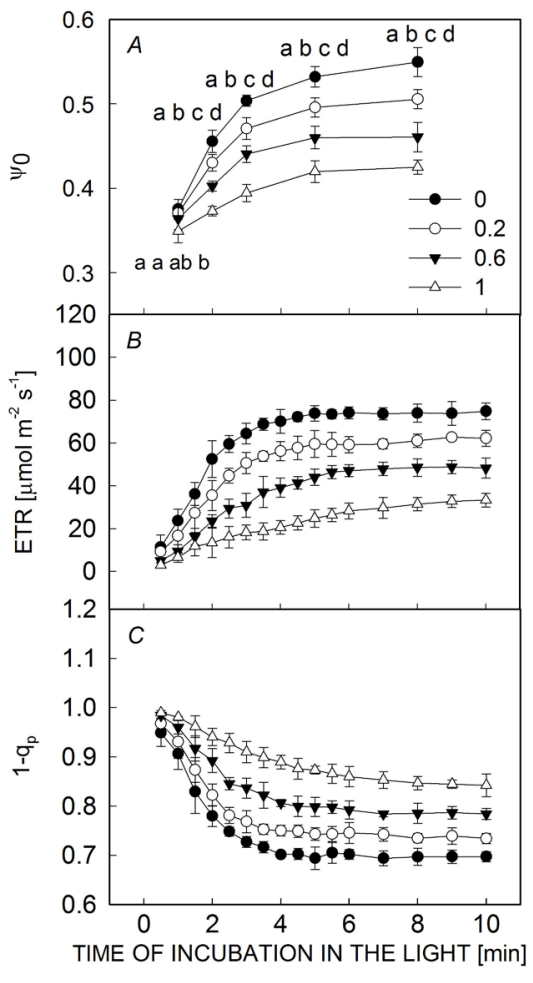

Fig. 3. (A) Exciting efficiency of electron transport beyond $\mathrm{Q}_{\mathrm{A}}^{-}\left(\Psi_{0}\right),(B)$ linear electron transport rate (ETR), and $(C)$ rate of closed PSII reaction centers $\left(1-\mathrm{q}_{\mathrm{P}}\right)$ in Rumex $\mathrm{K}-1$ leaves in the presence of 0 (control), $0.2,0.6$, or $1 \mathrm{mM}$ SHAM during photosynthetic induction. The lowercase letters represent values that differed significantly in the LSD range test $(P<0.05)$. Values are mean $\pm \mathrm{SE}, n=10$.

\section{Discussion}

Photosynthetic linear electron flow commonly increases more quickly than $\mathrm{CO}_{2}$ assimilation during photosynthetic induction (Zhou et al. 2004). In the present study, this phenomenon was indicated by the rapid response in $\Phi_{\text {PSII }}$ to sudden illumination (Fig. 3S, supplement). It raises two questions: (1) Is the mitochondrial AOX pathway, which acts as a photosynthetic electron sink during steady-state photosynthesis, also involved in the rapid response of photosynthetic linear electron flow to sudden illumination? (2) What are the physiological functions of rapid induction 
of AOX pathway-dependent photosynthetic linear electron flow during photosynthetic induction?

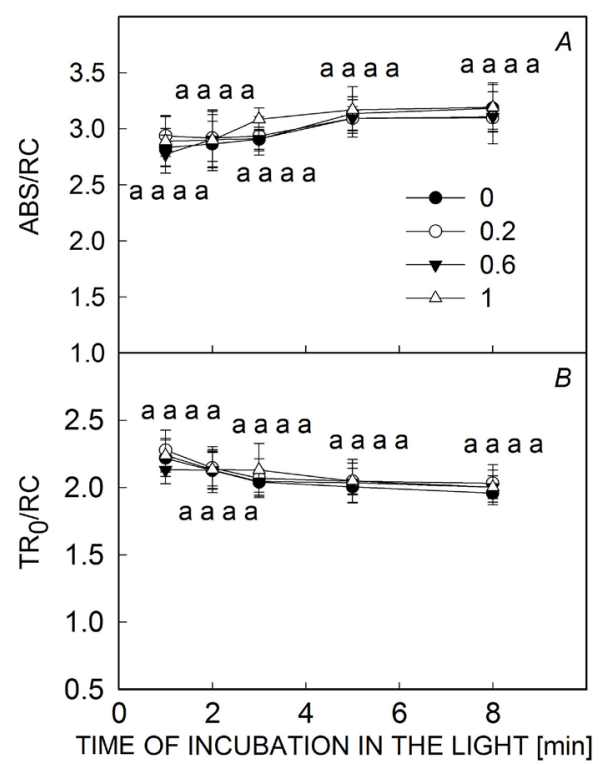

Fig. 4. (A) Light absorption per reaction center (ABS/RC) and $(B)$ light trapping per reaction center $\left(\mathrm{TR}_{0} / \mathrm{RC}\right)$ in Rumex $\mathrm{K}-1$ leaves in the presence of 0 (control), $0.2,0.6$, or 1 mM SHAM during photosynthetic induction. The lowercase letters represent values that differed significantly in the LSD range test $(P<0.05)$. Values are mean $\pm \mathrm{SE}, n=10$.

\section{The AOX pathway acts as a photosynthetic electron sink during photosynthetic induction}

Excess reducing equivalents generated by photosynthesis can be transported to other organelles via the malate-OAA shuttle and can be oxidized via the mitochondrial AOX pathway (Noguchi and Yoshida 2008, Selinski and Scheibe 2019, Zhao et al. 2020). Accordingly, the AOX pathway acts as an extra-chloroplastic sink for electrons generated during steady-state photosynthesis. NADP-MDH is a critical enzyme in the malate-OAA shuttle (Yokochi et al. 2021). Notably, the activation state of NADP-MDH increased rapidly when Rumex K-1 leaves were irradiated; almost $75 \%$ of full activation was reached only 1 min after irradiation (Fig. 2A). Thus, the malate-OAA shuttle was rapidly activated to export excess reducing equivalents generated by photosynthesis to mitochondria and cytosol during photosynthetic induction. In mitochondria and cytosol, internal and external $\mathrm{NAD}(\mathrm{P}) \mathrm{H}$ dehydrogenases can oxidize these reducing equivalents via the AOX pathway (Noguchi and Yoshida 2008).

In a previous study, when reducing equivalents accumulated in mitochondria, feedback led to downregulation in malate-OAA shuttle activity (Padmasree and Raghavendra 2001). Thus, it is reasonable to postulate that, during photosynthetic induction, the significant decrease in NADP-MDH activity after $1 \mathrm{~min}$ of illumination caused by the inhibition of the AOX was due to the accumulation of reducing equivalents in mitochondria, as consumption of reducing equivalents via the AOX pathway was restricted (Fig. 2A). A decrease in the activity of NADP-MDH
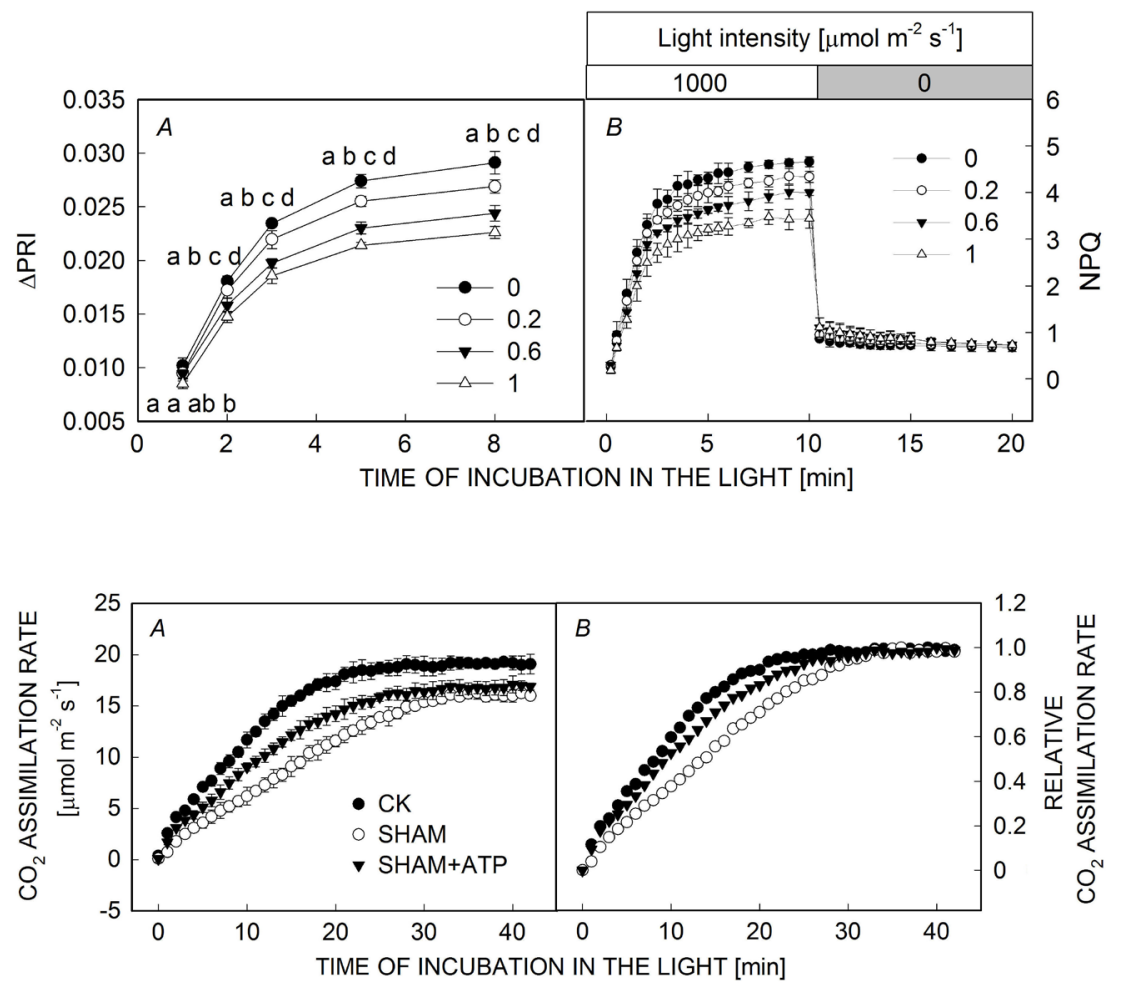

Fig. 5. (A) De-epoxidation of the xanthophyll cycle $(\triangle \mathrm{PRI})$ in Rumex $\mathrm{K}-1$ leaves in the presence of 0 (control), 0.2, 0.6, or $1 \mathrm{mM}$ SHAM during photosynthetic induction. (B) Nonphotochemical quenching (NPQ) in Rumex K-1 leaves in the presence of 0 (control), 0.2, 0.6, or $1 \mathrm{mM}$ SHAM during photosynthetic induction and dark relaxation. The lowercase letters represent values that differed significantly in the LSD range test $(P<0.05)$. Values are mean $\pm \mathrm{SE}, n=10$.

Fig. 6. (A) Curves showing light induction of $\mathrm{CO}_{2}$ assimilation in Rumex K-1 leaves treated with water (control), $1 \mathrm{mM}$ SHAM, or $1 \mathrm{mM} \mathrm{SHAM}+0.5 \mathrm{mM}$ ATP. (B) Relative $\mathrm{CO}_{2}$ assimilation rate during photosynthetic induction was derived from $(A): \mathrm{A}_{\mathrm{rt}}=\left(\mathrm{A}_{\mathrm{t}}-\mathrm{A}_{\min }\right) /\left(\mathrm{A}_{\max }-\mathrm{A}_{\min }\right)$, where $A_{r t}$ is the relative $\mathrm{CO}_{2}$ assimilation rate during photosynthetic induction at any time, $\mathrm{A}_{\mathrm{t}}$ is the $\mathrm{CO}_{2}$ assimilation rate during photosynthetic induction at any time, and $\mathrm{A}_{\max / \min }$ is the ratio of maximum/ minimum $\mathrm{CO}_{2}$ assimilation rates. Values are means $\pm \mathrm{SE}, n=5$. 
would inevitably limit the export of reducing equivalents from chloroplasts to mitochondria, causing accumulation of reducing equivalents in chloroplast stroma during photosynthetic induction. Thus, the reduction state of $\mathrm{P}_{700}$ was remarkably unregulated during photosynthetic induction (Fig. 2B), leading to overreduction of the PSI acceptor side during photosynthetic induction. As the increase in $\Psi_{0}$ was limited by SHAM treatment during photosynthetic induction (Fig. $3 A$ ), the increase in $\mathrm{P}_{700}$ reduction state due to inhibition of the AOX hindered electron transport from $\mathrm{Q}_{\mathrm{A}}$ to $\mathrm{Q}_{\mathrm{B}}$ (Strasser and Strasser 1995, Cheng et al. 2016a,b) and caused more severe reduction of the PSII acceptor side. Consequently, the proportion of closed PSII RCs $\left(1-\mathrm{q}_{\mathrm{P}}\right)$ increased (Fig. 3C), and photosynthetic linear ETR was hindered during photosynthetic induction (Fig. 3B).

Together, these results indicate that the AOX pathway also acts as a sink for electrons generated by photosynthesis during photosynthetic induction.

The AOX pathway protects the photosynthetic mechanism against photoinhibition during photosynthetic induction

The limitation of photosynthetic ETR (Fig. 3C) attributed to the inhibition of the AOX during photosynthetic induction resulted in two major events: (1) imbalance between light energy absorption and utilization, and (2) inhibition of NPQ formation.

Our results showed that SHAM treatments did not alter light absorption (Fig. 4A) or light trapping (Fig. 4B) per RC during photosynthetic induction, suggesting that chlorophyll antennae continuously absorbed much of the light energy, leading to charge separation that drives electron transport. Therefore, the limitation of photosynthetic ETR due to inhibition of the AOX inevitably causes a severe imbalance between photosynthetic light energy absorption and utilization during photosynthetic induction. Furthermore, to induce $\mathrm{q}_{\mathrm{E}}$ (the fast component of NPQ; xanthophyll cycle-dependent energy dissipation), a $\mathrm{pH}$ gradient across the thylakoid membrane $(\Delta \mathrm{pH})$ generated by photosynthetic linear and/or cyclic electron transport is required to activate de-epoxidation of violaxanthin to zeaxanthin (Miyake et al. 2005a,b; Hideg et al. 2008). In this study, de-epoxidation of the xanthophyll cycle, as indicated by $\triangle \mathrm{PRI}$, was limited by SHAM treatments (Fig. 5A), suggesting that the limitation in photosynthetic ETR due to the inhibition of the AOX also limited the generation of $\Delta \mathrm{pH}$ across the thylakoid membrane during photosynthetic induction. Thus, the development of NPQ was significantly suppressed (Figs. $5 B, 2 \mathrm{~S}$ ) because of AOX inhibition, which is consistent with the results from an Arabidopsis aoxla mutant reported by Zhang et al. (2010). In this study, the differences in NPQ between control and SHAM-treated leaves were eliminated within 1 min after being transferred from light to darkness (Fig. 5B), further indicating that suppression of NPQ development due to AOX inhibition was attributable to the suppression of $\mathrm{q}_{\mathrm{E}}$. The imbalance between light energy absorption and utilization and the suppression of
NPQ formation inevitably caused overexcitation of PSII RCs during photosynthetic induction, as indicated by upregulation of $1-\mathrm{q}_{\mathrm{P}}$ (Fig. $3 C$ ) caused by the inhibition of the AOX after illumination.

In chloroplasts, two major sites of reactive oxygen species (ROS) generation exist, i.e., the acceptor side of PSI and the RCs of PSII (Bartoli et al. 2005). Overreduction of the PSI acceptor side and overexcitation of PSII RCs due to inhibition of the AOX inevitably result in excess ROS production during photosynthetic induction, leading to photoinhibition. Therefore, acting as a photosynthetic electron sink, the AOX pathway is critical to photoprotection by preventing overreduction of the PSI acceptor side and overexcitation of PSII RCs during photosynthetic induction.

\section{The AOX pathway affects the activating process of Calvin cycle enzymes during photosynthetic induction}

The rate of activation of Calvin cycle enzymes (e.g., Rubisco) can hinder increased photosynthetic $\mathrm{CO}_{2}$ assimilation after the transfer from dark to light (Ruuska et al. 2000). Activation of Calvin cycle enzymes may require ATP hydrolysis (Makino et al. 2002). Compared with control leaves, induction of $\mathrm{CO}_{2}$ assimilation was slower in SHAM-treated leaves during photosynthetic induction (Fig. 6), suggesting that light activation of Calvin cycle enzymes was delayed because of AOX inhibition. Furthermore, induction of $\mathrm{CO}_{2}$ assimilation in SHAM-treated leaves was partially restored (through unknown mechanisms) by the application of exogenous ATP during photosynthetic induction (Fig. 6). These results indicate that the AOX pathway functions, at least partly, as a protector of ATP generation (the formation of $\Delta \mathrm{pH}$ across thylakoid membranes could be limited by the AOX pathway inhibition) to ensure Calvin cycle enzymes activation during photosynthetic induction. Other alternative electron sinks (e.g., the flow of cyclic electron transport) may supply ATP to activate Calvin cycle enzymes during photosynthetic induction (Makino et al. 2002). This raises the question: Why are several alternative reducing equivalent (or electron) sinks required during photosynthetic induction? The most likely reason for this apparent redundancy is that neither mechanism is completely effective.

Conclusion: When the AOX was suppressed during photosynthetic induction, the sink for excessive reducing equivalents generated by photosynthesis was reduced, which caused rapid accumulation of reducing equivalents (NADPH) in chloroplasts. Consequently, the PSI acceptor side was excessively reduced and $\mathrm{Q}_{\mathrm{A}}^{-}$accumulation increased. In such a scenario, the flow of photosynthetic linear electrons would be restricted, causing a further imbalance between light energy absorption and utilization, limiting the generation of thylakoid $\Delta \mathrm{pH}$, and promoting NPQ and ATP generation. Finally, inhibition of the AOX leads to severe photoinhibition and causes a delay in light activation of Calvin cycle enzymes (Fig. 7). Accordingly, the mitochondrial AOX pathway acts as a sink for electrons 


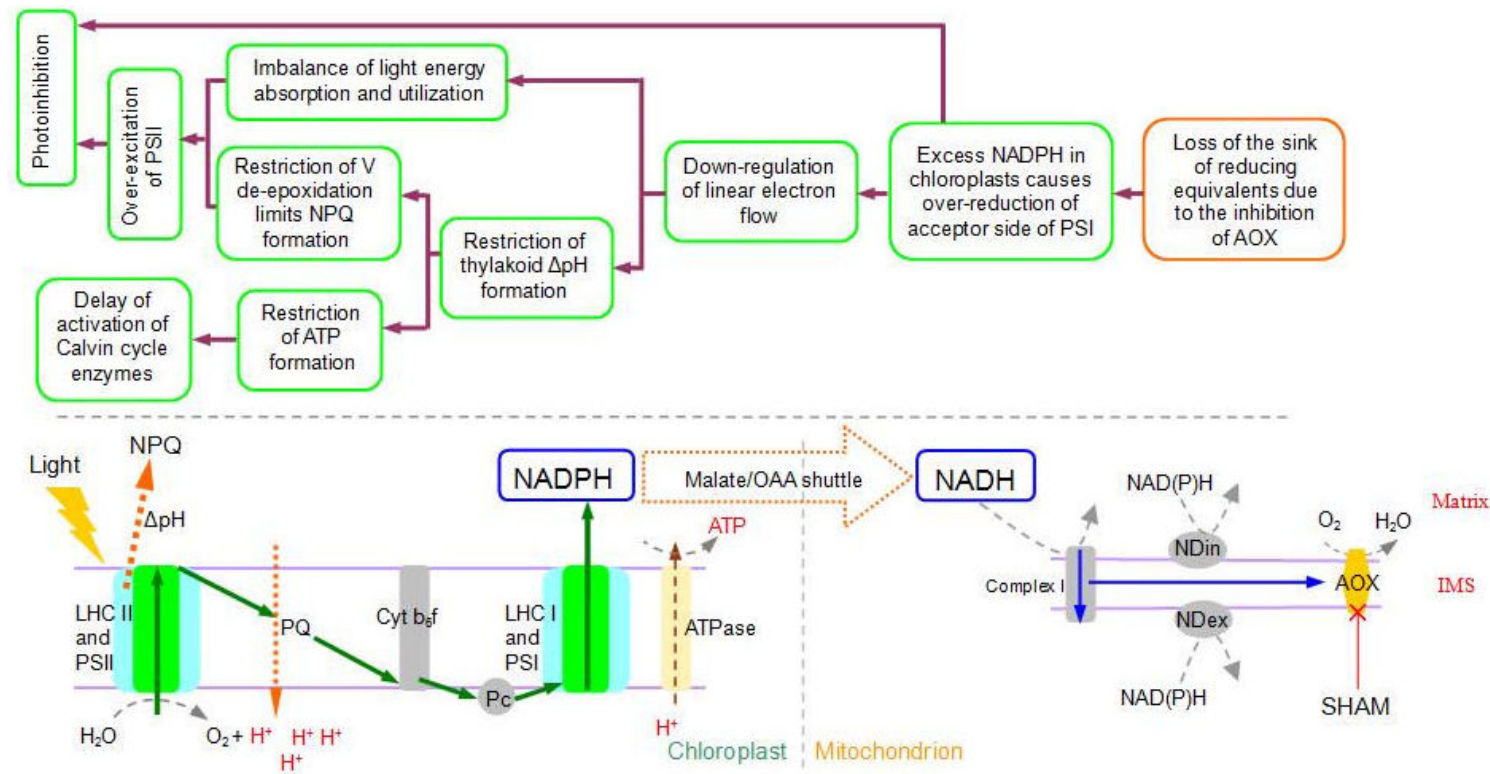

Fig. 7. Processes and likely states of cells during photosynthetic induction when the mitochondrial alternative oxidase (AOX) pathway is suppressed. LHCI/II - light-harvesting complex I/II; IMS - intermembrane space; NAD(P)-MDH - NAD(P)-malate dehydrogenase; NDex - external NAD(P)H-dehydrogenases; NDin - internal NAD(P)H-dehydrogenases; NPQ - nonphotochemical quenching; OAA - oxaloacetate; PSII/I - photosystem II/I; V - violaxanthin.

generated by photosynthesis, protecting the photosynthetic mechanism against photoinhibition and accelerating the induction of $\mathrm{CO}_{2}$ assimilation during photosynthetic induction.

\section{References}

Bartoli C.G., Gomez F., Gergoff G. et al:: Up-regulation of the mitochondrial alternative oxidase pathway enhances photosynthetic electron transport under drought conditions. J. Exp. Bot. 56: 1269-1276, 2005.

Betti M., Bauwe H., Busch F.A. et al.: Manipulating photorespiration to increase plant productivity: recent advances and perspectives for crop improvement. - J. Exp. Bot. 67: 2977 2988, 2016.

Chen H.X., An S.Z., Li W.J. et al.: Enhancement of the Mehlerperoxidase reaction in salt-stressed Rumex K-1 leaves. - Acta Bot. Sin. 46: 811-818, 2004.

Chen H.X., Li P.M., Gao H.Y.: Alleviation of photoinhibition by calcium supplement in salt-treated Rumex leaves. - Physiol. Plantarum 129: 386-396, 2007.

Cheng D.D., Liu M.J., Sun X.B. et al.: Light suppresses bacterial population through the accumulation of hydrogen peroxide in tobacco leaves infected with Pseudomonas syringae pv. tabaci. - Front. Plant Sci. 7: 512, 2016 a.

Cheng D.D., Zhang Z.S., Sun X.B. et al.: Photoinhibition and photoinhibition-like damage to the photosynthetic apparatus in tobacco leaves induced by Pseudomonas syringae pv. tabaci under light and dark conditions. - BMC Plant Biol. 16: $29,2016 b$.

Chivasa S., Carr J.P.: Cyanide restores $N$ gene-mediated resistance to tobacco mosaic virus in transgenic tobacco expressing salicylic acid hydroxylase. - Plant Cell 10: 14891498, 1998.

Dahal K., Martyn G.D., Vanlerberghe G.C.: Improved photo- synthetic performance during severe drought in Nicotiana tabacum overexpressing a nonenergy conserving respiratory electron sink. - New Phytol. 208: 382-395, 2015.

Dahal K., Vanlerberghe G.C.: Alternative oxidase respiration maintains both mitochondrial and chloroplast function during drought. - New Phytol. 213: 560-571, 2017.

Dahal K., Wang J., Martyn G.D. et al.: Mitochondrial alternative oxidase maintains respiration and preserves photosynthetic capacity during moderate drought in Nicotiana tabacum. Plant Physiol. 166: 1560-1574, 2014.

Del-Saz N.F., Ribas-Carbo M., McDonald A.E. et al.: An in vivo perspective of the role(s) of the alternative oxidase pathway. Trends Plant Sci. 23: 206-219, 2018.

Diethelm R., Miller M.G., Shibles R., Stewart C.R.: Effect of salicylhydroxamic acid on respiration, photosynthesis, and peroxidase activity in various plant tissues. - Plant Cell Physiol. 31: 179-185, 1990.

Dinakar C., Abhaypratap V., Yearla S.R. et al.: Importance of ROS and antioxidant system during the beneficial interactions of mitochondrial metabolism with photosynthetic carbon assimilation. - Planta 231: 461-474, 2010a.

Dinakar C., Raghavendra A.S., Padmasree K.: Importance of AOX pathway in optimizing photosynthesis under high light stress: role of pyruvate and malate in activating AOX. Physiol. Plantarum 139: 13-26, 2010b.

Driever S.M., Baker N.R.: The water-water cycle in leaves is not a major alternative electron sink for dissipation of excess excitation energy when $\mathrm{CO}_{2}$ assimilation is restricted. - Plant Cell Environ. 34: 837-846, 2011.

Dutilleul C., Driscoll S., Cornic G. et al.: Functional mitochondrial complex I is required by tobacco leaves for optimal photosynthetic performance in photorespiratory conditions and during transients. - Plant Physiol. 131: 264-275, 2003.

Fisher N., Meunier B., Biagini G.A.: The cytochrome $b c_{1}$ complex as an antipathogenic target. - FEBS Lett. 594: 29352952, 2020. 
Florez-Sarasa I., Noguchi K., Araújo W.L. et al.: Impaired cyclic electron flow around photosystem I disturbs high-light respiratory metabolism. - Plant Physiol. 172: 2176-2189, 2016.

Florez-Sarasa I., Ostaszewska M., Galle A. et al:: Changes of alternative oxidase activity, capacity and protein content in leaves of Cucumis sativus wild-type and MSC16 mutant grown under different light intensities. - Physiol. Plantarum 137: 419-426, 2009

Gamon J.A., Surfus J.S.: Assessing leaf pigment content and activity with a reflectometer. - New Phytol. 143: 105-117, 1999.

Heber U., Neimanis S., Lange O.L.: Stomatal aperture, photosynthesis and water fluxes in mesophyll cells as affected by the abscission of leaves. Simultaneous measurements of gas exchange, light scattering and chlorophyll fluorescence. Planta 67: 554-562, 1986.

Hideg É., Kós P.B., Schreiber U.: Imaging of NPQ and ROS formation in tobacco leaves: Heat inactivation of the waterwater cycle prevents down-regulation of PSII. - Plant Cell Physiol. 49: 1879-1886, 2008.

Hu W.H., Yan X.H., He Y., Ye X.L.: Role of alternative oxidase pathway in protection against drought-induced photoinhibition in pepper leaves. - Photosynthetica 56: 1297-1303, 2018.

Kirschbaum M.U.F., Pearcy R.W.: Gas exchange analysis of the relative importance of stomatal and biochemical factors in photosynthetic induction in Alocasia macrorrhiza. - Plant Physiol. 86: 782-785, 1988.

Laties G.G.: The cyanide-resistant, alternative path in plant mitochondria. - Ann. Rev. Plant Physio. 33: 519-555, 1982.

Li F.: [To devote major efforts to popularizing the Rumex forage grass and promote an adjustment of the agricultural structure.] - Sci. Tech. Rev. 18: 55-57, 2000. [In Chinese]

Makino A., Miyake C., Yokota A.: Physiological functions of the water-water cycle (Mehler reaction) and the cyclic electron flow around PSI in rice leaves. - Plant Cell Physiol. 43: 10171026, 2002.

Mathur S., Jain L., Jajoo A.: Photosynthetic efficiency in sun and shade plants. - Photosynthetica 56: 354-365, 2018.

Miller R.E., Grant N.M., Giles L. et al.: In the heat of the night - alternative pathway respiration drives thermogenesis in Philodendron bipinnatifidum. - New Phytol. 189: 10131026, 2011.

Miyake C., Horiguchi S., Makino A. et al.: Effects of light intensity on cyclic electron flow around PSI and its relationship to nonphotochemical quenching of $\mathrm{Chl}$ fluorescence in tobacco leaves. - Plant Cell Physiol. 46: 1819-1830, 2005a.

Miyake C., Miyata M., Shinzaki Y., Tomizawa K.: $\mathrm{CO}_{2}$ response of cyclic electron flow around PSI (CEF-PSI) in tobacco leaves-relative electron fluxes through PSI and PSII determine the magnitude of non-photochemical quenching (NPQ) of Chl fluorescence. - Plant Cell Physiol. 46: 629-637, 2005b.

Murphy A.M., Chivasa S., Singh D.P., Carr J.P.: Salicylic acidinduced resistance to viruses and other pathogens: a parting of the ways? - Trends Plant Sci. 4: 155-160, 1999.

Noguchi K., Yoshida K.: Interaction between photosynthesis and respiration in illuminated leaves. - Mitochondrion 8: 87-99, 2008.

Ogawa T., Sonoike K.: Dissection of respiration and photosynthesis in the cyanobacterium Synechocystis sp. PCC6803 by the analysis of chlorophyll fluorescence. - J. Photoch. Photobio. B 144: 61-67, 2015.

Omarova M.A., Artamonova N.A., Chasovitina G.M.: Chemical composition of the hybrid Rumex K-1. - Chem. Nat. Compd. 34: 426-428, 1998.

Padmasree K., Raghavendra A.S.: Consequence of restricted mitochondrial oxidative metabolism on photosynthetic carbon assimilation in mesophyll protoplasts: Decrease in light activation of four chloroplastic enzymes. - Physiol. Plantarum 112: 582-588, 2001

Rasmusson A.G., Fernie A.R., van Dongen J.T.: Alternative oxidase: a defence against metabolic fluctuations? - Physiol Plantarum 137: 371-382, 2009.

Robson C.A., Vanlerberghe G.C. Transgenic plant cells lacking mitochondrial alternative oxidase have increased susceptibility to mitochondria-dependent and -independent pathways of programmed cell death. - Plant Physiol. 129: 1908-1920, 2002.

Ruuska S.A., Bagder M.R., Andrews T.J., von Caemmerer S.: Photosynthetic electron sinks in transgenic tobacco with reduced amounts of Rubisco: little evidence for significant Mehler reaction. - J. Exp. Bot. 51: 357-368, 2000.

Scoffoni C., Sack L.: Are leaves 'freewheelin'? Testing for a wheeler-type effect in leaf xylem hydraulic decline. - Plant Cell Environ. 38: 534-543, 2015.

Selinski J., Scheibe R.: Malate valves: Old shuttles with new perspectives. - Plant Biol. 21: 21-30, 2019.

Smit M.F., van Heerden P.D.R., Pienaar J.J. et al: Effect of trifluoroacetate, a persistent degradation product of fluorinated hydrocarbons, on Phaseolus vulgaris and Zea mays. - Plant Physiol. Bioch. 47: 623-634, 2009.

Spicer R., Holbrook N.M.: Effects of carbon dioxide and oxygen on sapwood respiration in five temperate tree species. J. Exp. Bot. 58: 1313-1320, 2007.

Strasser B.J., Strasser R.J.: Measuring fast fluorescence transients to address environmental questions: The JIP test. - In: Mathis P. (ed.): Photosynthesis: From Light to Biosphere. Vol. 5. Pp. 977-980. Kluwer Academic Publishers, Dordrecht 1995.

Sui X.L., Sun J.L., Wang S.H. et al.: Photosynthetic induction in leaves of two cucumber genotypes differing in sensitivity to low-light stress. - Afr. J. Biotechnol. 10: 2238-2247, 2011.

Sunil B., Saini D., Bapatla R.B. et al.: Photorespiration is complemented by cyclic electron flow and the alternative oxidase pathway to optimize photosynthesis and protect against abiotic stress. - Photosynth. Res. 139: 67-79, 2019.

Sweetlove L.J., Lytovchenko A., Morgan M. et al.: Mitochondrial uncoupling protein is required for efficient photosynthesis. P. Natl. Acad. Sci. USA 103: 19587-19592, 2006.

Walker D.A.: The Use of the Oxygen Electrode and Fluorescence Probes in Simple Measurements of Photosynthesis. Pp. 212. The University of Sheffield, Sheffield 1988.

Watanabe C.K.A., Yamori W., Takahashi S. et al.: Mitochondrial alternative pathway-associated photoprotection of photosystem II is related to the photorespiratory pathway. - Plant Cell Physiol. 57: 1426-1431, 2016.

Yokochi Y., Yoshida K., Hahn F. et al.: Redox regulation of NADP-malate dehydrogenase is vital for land plants under fluctuating light environment. - P. Natl. Acad. Sci. USA 118: e2016903118, 2021.

Yoshida K., Terashima I., Noguchi K.: Up-regulation of mitochondrial alternative oxidase concomitant with chloroplast over-reduction by excess light. - Plant Cell Physiol. 48: 606614, 2007

Yoshida K., Watanabe C.K., Hachiya T. et al.: Distinct responses of the mitochondrial respiratory chain to long- and short-term high-light environments in Arabidopsis thaliana. - Plant Cell Environ. 34: 618-628, 2011.

Yusuf M.A., Kumar D., Rajwanshi R. et al.: Overexpression of $\gamma$-tocopherol methyl transferase gene in transgenic Brassica juncea plants alleviates abiotic stress: Physiological and chlorophyll $a$ fluorescence measurements. - BBA- 
Bioenergetics 1797: 1428-1438, 2010.

Zabalza A., van Dongen J.T., Froehlich A. et al.: Regulation of respiration and fermentation to control the plant internal oxygen concentration. - Plant Physiol. 149: 1087-1098, 2009.

Zhang D.W., Xu F., Zhang Z.W. et al.: Effects of light on cyanide-resistant respiration and alternative oxidase function in Arabidopsis seedlings. - Plant Cell Environ. 33: 21212131, 2010.

Zhang Z.S., Liu M.J., Scheibe R. et al.: Contribution of the alternative respiratory pathway to PSII photoprotection in $\mathrm{C}_{3}$ and $\mathrm{C}_{4}$ plants. - Mol. Plant 10: 131-142, 2017.

Zhao Y., Yu H., Zhou J. et al.: Malate circulation: Linking chloroplast metabolism to mitochondrial ROS. - Trends Plant Sci. 25: 446-454, 2020.

Zhou Y.H., Yu J.Q., Huang L.F., Nogués S.: The relationship between $\mathrm{CO}_{2}$ assimilation, photosynthetic electron transport and water-water cycle in chill-exposed cucumber leaves under low light and subsequent recovery. - Plant Cell Environ. 27: 1503-1514, 2004.

(C) The authors. This is an open access article distributed under the terms of the Creative Commons BY-NC-ND Licence. 